\title{
FERTILIDADE, MATÉRIA ORGÂNICA E SUBSTÂNCIAS HÚMICAS EM SOLOS ANTROPOGÊNICOS DA AMAZÔNIA OCIDENTAL $\left({ }^{1}\right)$
}

\author{
ADÔNIS MOREIRA $\left({ }^{2}\right)$
}

\begin{abstract}
RESUMO
O objetivo deste trabalho foi avaliar as alterações químicas e as variações no estoque e na qualidade da matéria orgânica em solos de alta fertilidade de formação antropogênica, denominado Terra Preta do Índio (TPI), existente na Amazônia. Para isso, foram utilizadas 21 amostras de terra com diferentes profundidades, provenientes de onze sítios arqueológicos e de quatro classes de solos da região (Neossolo, Latossolo, Argissolo e Cambissolo). O caráter eutrófico da TPI contrasta com os solos representativos da região e, mesmo com a alta fertilidade, observam-se nos sítios grande heterogeneidade nos teores de $\mathrm{N}$ total, C orgânico, P orgânico e disponível, K disponível, Ca e Mg trocável. A ação antrópica pré-colombiana nos solos da Amazônia ocasionou em aumento do conteúdo de ácidos húmicos e humina e redução dos ácidos fúlvicos, com conseqüente melhoria na qualidade da matéria orgânica do solo. O método WalkleyBlack tem limitações na determinação do carbono nos solos antropogênicos quando comparado com analisador CHNS.
\end{abstract}

Palavras-chave: ácidos fúlvicos, ácidos húmicos, humina, Terra Preta do Índio, C orgânico, $\mathrm{N}$ total.

\section{ABSTRACT \\ SOIL FERTILITY, ORGANIC MATTER AND HUMIC COMPOUNDS IN ANTHROPOGENIC SOILS OF WESTERN AMAZON}

The objective of this work was to evaluate the variations of the carbon stock and quality and the chemistry alterations of soils of anthropogenic formation with high fertility occurring in scattered patches throughout the Amazon, called "Terra Preta do Índio" (Amazonian Dark Earths - ADE). Twenty-one samples of different depths taken from eleven archeology sites, and four representative soil classes of the region (Oxisol, Inceptsol, Ultisol and Spodsol) were used. The eutrophic character of Amazonian Dark Earths contrast with these representative soils and despite its high fertility, the sites present a great heterogeneity concerning the concentration of total $\mathrm{N}$, organic $\mathrm{C}$, available and organic $\mathrm{P}$, available $\mathrm{K}$ and exchangeable $\mathrm{Ca}$ and $\mathrm{Mg}$. The ancient antropic action in Amazonian soils resulted in increase of humic acids and humin content and decrease of the fulvic acids, with consequent improvement of the soil organic matter quality. The use of Walkley-Black method presented limitations for the carbon in the anthropogenic soils as compared to the CHNS analyzer.

Key words: fulvic acid, humic acid, humin, Amazonian Dark Earths, organic C, total N.

$\left({ }^{1}\right)$ Recebido para publicação em 27 de dezembro de 2005 e aceito em 26 de dezembro de 2006

$\left({ }^{2}\right)$ Embrapa Pecuária Sudeste, Caixa Postal 339, 13560-970 São Carlos (SP). Bolsista do CNPq. E-mail: adonis@cppse.embrapa.br. 


\section{INTRODUÇÃO}

Apesar da existência de grandes áreas potencialmente agricultáveis, os solos da Amazônia, na sua maior parte, são ácidos, com baixa capacidade de troca catiônica e, conseqüentemente, baixa fertilidade (Moreira e Malavolta, 2002). Dos poucos solos com alta fertilidade, incluem-se a "Terra Preta do Índio" (TPI), denominação dada aos solos de origem antropogênica, existentes na Região Amazônica (Smith, 1980; Woods, 2003). Esses solos, geralmente, estão localizados próximo dos rios e na sua maioria são distribuídos em áreas de dois a cinco hectares (TEIXEIRA e MARTINS, 2003), com elevados níveis de nutrientes, principalmente $\mathrm{Ca}$ e $\mathrm{P}$, altos teores de matéria orgânica e atividade biológica mais elevada que os solos adjacentes (STEINER et al., 2004a), na sua maioria cauliníticos e fortemente intemperizados.

A TPI é caracterizada por um horizonte A antrópico, de cor escura, com alto teor de matéria orgânica. Muitos aspectos de sua origem ainda não estão elucidados, tendo sido formado, provavelmente, pelo homem pré-colombiano e abandonado depois da invasão dos europeus (KERn e KäMPf, 1989). Porém, muitas dúvidas permanecem, entre elas destaca-se a alta capacidade de manutenção da fertilidade com o uso contínuo da terra, mesmo nas condições edafoclimáticas locais.

Essa capacidade de manter o alto teor de carbono orgânico ocorre, possivelmente, devido às características químicas e da resistência do material à decomposição microbiana. A matéria orgânica do solo (MOS) é contituída, em sua maior parte, por substâncias húmicas mais estáveis, de difícil degradação. Essas substâncias são formadas a partir da transformação dos resíduos orgânicos realizada pela biomassa microbiana presente no solo e pela polimerização dos compostos orgânicos processados até a síntese de macromoléculas resistentes à degradação biológica (CAMARGo et al., 1999).

Segundo Stevenson (1994), as substâncias húmicas (humina, ácidos fúlvicos e ácidos húmicos) são consideradas a parte final da evolução da MOS e representam cerca de $70 \%$ do C presente no solo; são diferenciadas, principalmente, através dos grupos funcionais (fenólicos, carboxílicos, entre outros) e grau de polimerização. Os ácidos húmicos são insolúveis em meio ácido e solúveis em meio básico e possuem estrutura grande ( $8 \AA$ ) e complexa, quando comparados com os ácidos fúlvicos, completamente hidrossolúveis, com tamanho pequeno $(2 \AA)$, maiores grupamentos carboxílicos e de oxigênio e menor concentração de C, favorecendo sua percolação no solo. No caso da humina, esta é insólúvel em meio ácido e básico e tem maior grau de polimerização que os ácidos fúlvicos e húmicos (PRIMAVEsI, 1990; SCHNITZER et al., 1991).
O objetivo deste trabalho foi avaliar as alterações no estoque de carbono orgânico e solúvel em água, na fertilidade e nas substâncias húmicas (ácidos fúlvicos, ácidos húmicos e humina) do solo decorrentes das atividades antrópicas précolombianas, comparado-as com quatro classes de solos representativas da Região Amazônica.

\section{MATERIAL E MÉTODOS}

Foram analisadas em duplicata, 21 amostras de terra provenientes de diferentes profundidades originárias de onze sítios arqueológicos, sendo eles: Costa do Açutuba, nas margens do rio Negro, Município de Iranduba (AM); Hatahara, nas margens do rio Solimões, Município de Iranduba (AM); Caldeirão, nas margens do rio Solimões, Município de Iranduba (AM); Jiquitaia, nas margens do rio Preto da Eva, Município de Rio Preto da Eva (AM); Costa do Laranjal, nas margens do rio Solimões, Município de Manacapuru (AM); Itapeaçu, nas margens do rio Amazonas, Município de Urucurituba (AM); Cajutuba, nas margens do rio Tapajós, Município de Santarém (PA); Caburi, nas margens do rio Amazonas, Município de Parintins (AM); Serra Baixa, nas margens do rio Solimões, Município de Manacapuru (AM); Rio Urubu, na margem do rio Urubu, Município de Rio Preto da Eva (AM) e Terra Preta do Limão, nas margens do rio Amazonas, Município de Barreirinha (AM). Concomitantemente, em nível de comparação, também foram analisadas quatro amostras de classes de solos representativas da região, sendo: o Neossolo Flúvico, coletado no Município de Presidente Figueiredo (AM), o Latossolo Amarelo, coletado no Município de Manaus (AM), o Argissolo Amarelo, coletado no Município de Rio Preto da Eva (AM) e o Cambissolo Háplico, coletado no Município de Coari (AM).

Nas amostras estudadas (Tabela 1), foram analisadas o $\mathrm{pH}, \mathrm{P}$ disponível, $\mathrm{K}$ disponível, $\mathrm{Ca}$ trocável, $\mathrm{Mg}$ trocável, Al trocável e a acidez potencial (EmbrapA, 1997). O pH foi determinado em $\mathrm{CaCl}_{2} 0,01$ mol $\mathrm{L}^{-1}$ na relação $1: 2,5$; o $\mathrm{P}$ disponível e o $\mathrm{K}$ disponível foram extraídos por Mehlich 1 (0,025 mol $\mathrm{L}^{-1}$ de $\mathrm{H}_{2} \mathrm{SO}_{4}+0,05$ mol L ${ }^{-1}$ de $\left.\mathrm{HCl}\right)$. O Ca, $\mathrm{Mg}$ e $\mathrm{Al}$ trocável foram extraídos por $\mathrm{KCl} \mathrm{1,0} \mathrm{mol} \mathrm{L}^{-1}$. A acidez potencial $(\mathrm{H}+\mathrm{Al})$ foi determinada indiretamente por meio da solução-tampão SMP (MoreIrA et al., 2004). O C orgânico foi determinado pelo método WalkleyBlack (EMbrapA, 1997), e também pelo método de oxidação seca com o analisador CHNS. O N total foi obtido através do analisador CHNS, enquanto o $\mathrm{P}$ orgânico foi analisado de acordo com método descrito por Olsen e SOMMERS (1982). 
Tabela 1. Análise química dos solos estudados e estatística descritiva

\begin{tabular}{|c|c|c|c|c|c|c|c|c|c|c|}
\hline Sítio & Localização & Prof & $\mathrm{pH}$ & $\mathrm{P}$ & K & $\mathrm{Ca}$ & $\mathrm{Mg}$ & $\mathrm{Al}$ & $\mathrm{H}+\mathrm{Al}$ & $\mathrm{V}$ \\
\hline & & $\mathrm{cm}$ & $\mathrm{CaCl}_{2}$ & \multicolumn{2}{|c|}{$\mathrm{mg} \mathrm{dm}^{-3}$} & 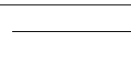 & $-\mathrm{mmol}_{\mathrm{c}}$ & $\mathrm{dm}^{-3}$ & & $\%$ \\
\hline Rio Urubu & R. Preto da Eva (AM) & $0-10$ & 4,4 & 268,9 & 40,0 & 95,0 & 63,0 & 1,0 & 60,0 & 72,6 \\
\hline Rio Urubu & R. Preto da Eva (AM) & $0-20$ & 3,9 & 343,0 & 4,0 & 10,0 & 1,0 & 6,0 & 38,0 & 22,4 \\
\hline Hatahara & Iranduba (AM) & $0-20$ & 4,8 & 1093,2 & 183,0 & 109,0 & 41,0 & 1,0 & 21,0 & 88,0 \\
\hline Hatahara & Iranduba (AM) & $20-40$ & 4,3 & 1060,7 & 37,0 & 33,0 & 23,0 & 1,0 & 41,0 & 72,8 \\
\hline Hatahara & Iranduba (AM) & $40-60$ & 4,4 & 1371,6 & 41,0 & 73,0 & 19,0 & 1,0 & 35,0 & 72,6 \\
\hline Serra Baixa & Manacapuru (AM) & $0-20$ & 4,2 & 8,6 & 8,0 & 1,0 & 1,0 & 6,0 & 31,0 & 6,6 \\
\hline Jiquitaia & R. Preto da Eva (AM) & $0-20$ & 4,1 & 117,0 & 12,0 & 27,0 & 13,0 & 1,0 & 40,0 & 50,0 \\
\hline Jiquitaia & R. Preto da Eva (AM) & $20-40$ & 4,3 & 254,4 & 26,0 & 35,0 & 54,0 & 1,0 & 43,0 & 67,6 \\
\hline Açutuba & Iranduba (AM) & $0-20$ & 4,0 & 86,3 & 16,0 & 13,0 & 2,0 & 2,0 & 25,0 & 38,1 \\
\hline Jiquitaia & R. Preto da Eva (AM) & $0-10$ & 4,4 & 1208,9 & 75,0 & 32,0 & 16,0 & 1,0 & 26,0 & 65,7 \\
\hline C. Laranjal & Manacapuru (AM) & $0-20$ & 4,3 & 549,1 & 12,0 & 23,0 & 20,0 & 1,0 & 27,0 & 61,6 \\
\hline Caburi & Parintins (AM) & $0-20$ & 4,1 & 939,6 & 22,0 & 52,0 & 2,0 & 8,0 & 93,0 & 37,0 \\
\hline Cajutuba & Santarém (PA) & $0-20$ & 3,9 & 936,0 & 22,0 & 9,0 & 1,0 & 75,0 & 178,0 & 1,4 \\
\hline Itapeaçu & Urucurituba (AM) & $0-20$ & 4,0 & 292,4 & 58,0 & 131,0 & 28,0 & 2,0 & 92,0 & 63,6 \\
\hline Caldeirão & Iranduba (AM) & $0-20$ & 4,7 & 339,4 & 38,0 & 238,0 & 56,0 & 1,0 & 84,0 & 77,8 \\
\hline Barreirinha & Barreirinha (AM) & $0-20$ & 4,4 & 264,4 & 137,2 & 102,0 & 27,0 & 1,0 & 134,0 & 49,7 \\
\hline C. Laranjal & Manacapuru (AM) & $0-10$ & 4,8 & 751,6 & 38,0 & 314,0 & 32,0 & 1,0 & 66,0 & 84,0 \\
\hline Média & & - & 4,3 & 581,5 & 45,3 & 82,0 & 23,0 & 6,0 & 61,0 & 54,8 \\
\hline Mediana & & - & 4,3 & 343,0 & 37,0 & 35,0 & 21,0 & 1,0 & 41,0 & 61,1 \\
\hline Desvio padrão & & - & 0,3 & 439,3 & 47,6 & 90,0 & 20,0 & 18,0 & 44,0 & 27,3 \\
\hline Assimetria & & - & ,29 & 0,40 & 2,02 & 1,41 & 0,56 & 3,91 & 1,48 & 0,71 \\
\hline Curtose & & - & $-0,63$ & $-1,41$ & 3,87 & 1,48 & $-06,3$ & 15,46 & 1,93 &,- 040 \\
\hline Shapiro Wilks $\left({ }^{1}\right)$ & & - & $0,94^{\mathrm{NS}}$ & $0,89^{\mathrm{NS}}$ & $0,74^{*}$ & $0,83^{*}$ & $0,92^{\mathrm{NS}}$ & $0,35^{*}$ & $0,83^{*}$ & $0,71^{*}$ \\
\hline CV (\%) & & - & 7,52 & $75,54 \quad 1$ & 105,28 & 109,21 & $87,46 \quad 2$ & 284,95 & 71,34 & 50,39 \\
\hline Neossolo & P. Figueiredo (AM) & $0-20$ & 4,3 & 11,5 & 6,0 & 0,4 & 0,4 & 6,0 & 28,0 & 3,3 \\
\hline Argissolo & R. Preto da Eva (AM) & $0-20$ & 4,0 & 2,0 & 13,0 & 0,3 & 0,4 & 9,0 & 18,0 & 5,3 \\
\hline Cambissolo & Coari (AM) & $0-10$ & 4,4 & 2,0 & 2,0 & 0,3 & 0,4 & 5,0 & 17,0 & 5,6 \\
\hline Latossolo & Manaus (AM) & $0-20$ & 3,5 & 2,0 & 21,0 & 0,5 & 1,0 & 21,0 & 49,0 & 3,9 \\
\hline Média & & - & 4,1 & 4,4 & 10,5 & 0,3 & 0,6 & 10,0 & 28,0 & 4,5 \\
\hline Mediana & & - & 4,5 & 2,0 & 9,5 & 0,3 & 0,4 & 74,0 & 23,0 & 4,6 \\
\hline Desvio padrão & & - & 0,4 & 4,8 & 8,4 & 0,1 & 0,3 & 8,0 & 15,0 & 1,1 \\
\hline $\mathrm{CV}(\%)$ & & - & 9,8 & 109,1 & 80,0 & 33,3 & 50,0 & 80,0 & 53,6 & 24,4 \\
\hline
\end{tabular}

$\left({ }^{1}\right)$ *Significativo a $5 \%$ de probabilidade. ${ }^{\text {NS }}$ : Não-significativo.

Para extração e fracionamento químico da matéria orgânica [C solúvel em água - CSA, matérias húmicas (ácidos fúlvicos - CAF, ácidos húmicos $\mathrm{CAH}$ ) e humina - $\mathrm{CHU}$, foi utilizado o método adaptado de Schnitzer (1982), que consiste nos seguintes procedimentos: $10 \mathrm{~g}$ de TFSA (Terra Fina Seca ao Ar) colocados em tubo de centrífuga com 50 $\mathrm{mL}$ de água desionizada e agitados por 30 minutos e centrifugados por 20 minutos, a $13232 \mathrm{~g}$. Posteriormente, transferiu-se o sobrenadante para um frasco (C solúvel em água). O material retido no tubo foi ressuspendido com $\mathrm{NaOH} 0,1 \mathrm{~mol} \mathrm{~L}^{-1} \mathrm{em}$ agitador horizontal por uma hora e centrifugado por 12 minutos a $10131 \mathrm{~g}$. Em seqüência, $100 \mathrm{~mL}$ do sobrenadante (matérias húmicas) foram novamente colocados no tubo

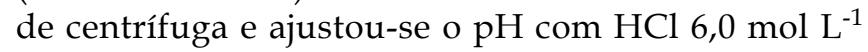
até 2,0, deixando a solução em repouso por 30 minutos e, posteriormente centrifugando por 10 minutos a $5168 \mathrm{~g}$. O sobrenadante (ácidos fúlvicos) foi transferido em frascos e o precipitado (ácidos húmicos) foi ressuspendido com $25 \mathrm{~mL}$ de $\mathrm{NaOH} 0,1 \mathrm{~mol} \mathrm{~L}^{-1}$. A fração humina foi calculada pela diferença entre o $\mathrm{C}$ orgânico (Walkley-Black) e C encontrado na fração matérias húmicas. 
As análises descritivas (média, mediana, desvio-padrão, coeficientes de curtose, assimetria e de variação) dos atributos químicos do solo e do fracionamento da matéria orgânica foram realizadas de acordo com Ribeiro JúNIOR (1995). Na comparação dos dados foram realizadas as análises de variância (Teste F) e correlações a 5 \% de significância (GOMES e Garcia, 2002). Para verificar a existência de distribuição normal dos atributos do solo nos sítios amostrados foi utilizado o teste não-paramétrico de Shapiro Wilks a 5\% de significância.

\section{RESULTADOS E DISCUSSÃO}

Os valores de $\mathrm{pH}, \mathrm{P}$ e $\mathrm{Mg}$ nos solos antropogênicos foram de média e mediana semelhança e comportamento tendendo à normalidade. $\mathrm{O}$ mesmo não foi observado com os de $\mathrm{K}, \mathrm{Ca}, \mathrm{Al}$ e $\mathrm{H}+\mathrm{Al}$, que tiveram distribuição lognormal, com coeficientes de assimetria positivos variando de 1,41 a 3,91, o que caracteriza haver uma elevada freqüência de valores abaixo da média (Tabela 1). As diferenças observadas dentro da TPI, provavelmente, são consequiências das suas localizações, haja vista que nos sítios localizados nas proximidades dos rios de água barrenta (maior quantidade de sedimentos), como o Solimões e o Madeira, verificaram-se maior fertilidade natural quando comparados com os localizados às margens dos rios de águas escuras ou claras, como os rios Negro e Tapajós.

Adotando os critérios de classificação de Gomes e GARCia (2002) para comparar a dispersão dos dados através dos coeficientes de variação, verificou-se no $\mathrm{pH}$ os menores valores, enquanto os maiores coeficientes foram observados no $\mathrm{Al}$ trocável (Tabela 1). Independentemente do tipo de solo ser de origem antrópica ou não, os coeficientes verificados nos teores de $\mathrm{P}, \mathrm{K}, \mathrm{Ca}, \mathrm{Mg}$, $\mathrm{Al}$ e $\mathrm{H}+\mathrm{Al}$ ficaram dentro das classes alta $(20 \%$ a $30 \%)$ e muito alta $(>30 \%)$. Esses altos coeficientes de variação corroboram os de Coelho (2003), sobre a presença de alta variabilidade nesses atributos do solo devido ao manejo adotado, da dependência dos processos de formação e do balanço no espaço e no tempo.

Os valores de $\mathrm{pH}$ em $\mathrm{CaCl}_{2}$ 0,01 $\mathrm{mol} \mathrm{L}^{-1}$ variaram de 3,9 (Rio Urubu e Cajutuba, 0-20 cm) a 4,8 (Hatahara e Costa do Laranjal, $0-20 \mathrm{~cm}$ ), ficando na média, em 4,3 ; valores de, $0,8(23 \%)$ e $0,4(7,0 \%)$ unidades superiores aos Latossolos e Argissolos, juntamente, representam aproximadamente $75 \%$ dos solos da região (Tabela 1). Segundo RAIJ et al. (1997), esses resultados estão dentro das classes de interpretação considerada baixa e muito baixa, para o Estado de São Paulo.
Exceto o sítio de TPI Serra Baixa, com $8,6 \mathrm{mg}$ $\mathrm{dm}^{-3}$ de $\mathrm{P}$ disponível e $1,0 \mathrm{mmol}_{\mathrm{c}} \mathrm{dm}^{-3}$ de Ca trocável, a exemplo do que foi mostrado na revisão realizada por LeHMANN et al. (2003), os solos antropogênicos possuem, com algumas exceções, altos teores de $\mathrm{P}$ disponível e Ca trocável (Tabela 1), variando de $86,3 \mathrm{mg} \mathrm{dm}^{-3}$ e $9,0 \mathrm{mmol}_{\mathrm{c}} \mathrm{dm}^{-3}$ nos sítios Açutuba (Iranduba) e Cajutuba (Santarém), respectivamente $(0-20 \mathrm{~cm})$ a $1371,6 \mathrm{mg} \mathrm{dm}^{-3}$ e 238,0 $\mathrm{mmol}_{\mathrm{c}} \mathrm{dm}^{-3}$ nos sítios Hatahara e Caldeirão (40-60 $\mathrm{cm}$ e $0-20 \mathrm{~cm}$ ). Esses valores são superiores aos observados, na média dos outros solos representativos da Amazônia $\left(4,4 \mathrm{mg} \mathrm{dm}^{-3}\right)$, e dos definidos por Alvarez Venegas et al. (1999) na classe de interpretação Bom $\left(\mathrm{P}=8,1\right.$ a $12 \mathrm{mg} \mathrm{dm}^{-3} \mathrm{e}$ $\mathrm{Ca}=0,36$ a $\left.0,6 \mathrm{mmol}_{\mathrm{c}} \mathrm{dm}^{-3}\right)$, utilizando os extratores Mehlich 1 e $\mathrm{KCl} 1,0 \mathrm{~mol} \mathrm{L^{-1 }}$ respectivamente. Verificou-se, também, que $27,3 \%$ das amostras de TPI não tinham os teores de $\mathrm{P}$ disponível ${ }^{3} 180 \mathrm{mg} \mathrm{dm}^{-3}$ (extrator Mehlich 1) proposto por KERN e KÄMPF (1989) como um dos requisitos para classificação dos solos antropogênicos.

As diferenças observadas nos teores de $\mathrm{P}$ disponível e Ca trocável entre os sítios amostrados podem ser atribuídas ao uso agrícola intensivo atual, às perdas por erosão superficial (os produtores não fazem nenhuma prática de manejo de conservação do solo), localização (Lima et al., 2002) e ao tamanho e período de residência das populações indígenas nas localidades (SJOBERG, 1976). A variação nos teores desses elementos em profundidade foi, possivelmente, decorrente da lixiviação ocasionada pela alta precipitação pluvial da região (KERN e KäMPF, 1989). Com relação ao P disponível, essa lixiviação também pode ser atribuída à alta quantidade de carbono orgânico (Tabela 2) e, conseqüentemente, de ânions orgânicos, reduzindo a adsorção de $\mathrm{P}$ à caulinita favorecendo sua translocação ao longo do perfil do solo (LopezHernandeZ et al., 1986).

A média dos teores de $\mathrm{Mg}$ trocável na TPI também está acima de $20,0 \mathrm{mmol}_{\mathrm{c}} \mathrm{dm}^{-3}$, situando dentro da faixa considerada muito boa, enquanto os teores trocáveis de $\mathrm{Al}$ e de $\mathrm{H}+\mathrm{Al}\left(6,0 \mathrm{mmol}_{\mathrm{c}} \mathrm{dm}^{-3}\right.$ e $\left.61,0 \mathrm{mmol}_{\mathrm{c}} \mathrm{dm}^{-3}\right)$ ficaram, respectivamente, dentro das classes média e alta, (Alvarez Venegas et al., 1999). Exceto o K disponível, com baixos teores, o que concorda com o observado por LEHMANN et al. (2003), os demais atributos analisados são contrastantes com os solos representativos da região, que se caracterizam por ter baixa fertilidade natural (Tabela 1), com os sítios de troca ocupados quase exclusivamente por hidrogênio e alumínio (Demattê, 1988). 
Os resultados do teste de normalidade de Shapiro Wilks $(p £ 0,05)$ indicaram distribuição normal para os teores de C-CHNS, P orgânico e relação $\mathrm{C} / \mathrm{N}$ (Tabela 2); o mesmo não ocorreu com o $\mathrm{N}$ total, com desvio-padrão e coeficiente de variação altos, valores de mínimo e máximo variando de 0,58 $\mathrm{g} \mathrm{kg}^{-1}$ no sítio arqueológico do Açutuba (Iranduba) a $5,19 \mathrm{~g} \mathrm{~kg}^{-1}$ no Cajutuba (Santarém). Apesar dos baixos teores verificados nos solos, os teores de $\mathrm{N}$ total indicaram correlação positiva e significativa com o carbono orgânico determinado pelo método WalkleyBlack (Figura 1).

Tabela 2. Carbono - WB (Walkley \& Black), carbono e nitrogênio total (analisador CHNS), relação C/N e P orgânico nos solos estudados e estatística descritiva

\begin{tabular}{|c|c|c|c|c|c|c|c|}
\hline Sítio & Localização & Prof & $C-W B$ & C - CHNS & $\mathrm{N}$ & $\mathrm{C} / \mathrm{N}$ & P org \\
\hline & & $\mathrm{cm}$ & 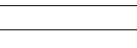 & $\mathrm{kg}^{-1}$ & & & $\mathrm{mg} \mathrm{kg}^{-1}$ \\
\hline Rio Urubu & R, Preto da Eva (AM) & $0-10$ & 44,97 & 76,20 & 3,00 & 25,39 & 316,53 \\
\hline Rio Urubu & R, Preto da Eva (AM) & $0-20$ & 10,23 & 20,50 & 0,85 & 24,08 & 477,30 \\
\hline Hatahara & Iranduba (AM) & $0-20$ & 30,69 & 27,20 & 1,63 & 16,67 & 808,85 \\
\hline Hatahara & Iranduba (AM) & $20-40$ & 28,41 & 25,60 & 1,18 & 21,66 & 768,55 \\
\hline Hatahara & Iranduba (AM) & $40-60$ & 23,82 & 21,80 & 0,90 & 24,26 & 987,63 \\
\hline Serra Baixa & Manacapuru (AM) & $0-20$ & 15,48 & 11,10 & 0,65 & 17,08 & 30,38 \\
\hline Jiquitaia & R, Preto da Eva (AM) & $0-20$ & 25,39 & 23,30 & 1,50 & 15,51 & 167,97 \\
\hline Jiquitaia & R, Preto da Eva (AM) & $20-40$ & 36,93 & 36,80 & 2,19 & 16,80 & 557,80 \\
\hline Açutuba & Iranduba (AM) & $0-20$ & 9,36 & 8,90 & 0,58 & 15,41 & 62,00 \\
\hline Jiquitaia & R, Preto da Eva (AM) & $0-10$ & 21,51 & 19,30 & 1,14 & 16,95 & 803,12 \\
\hline C. Laranjal & Manacapuru (AM) & $0-20$ & 17,65 & 12,50 & 0,83 & 15,08 & 397,99 \\
\hline Caburi & Parintins (AM) & $0-20$ & 16,35 & 13,90 & 0,93 & 15,01 & 335,37 \\
\hline Cajutuba & Santarém (PA) & $0-20$ & 33,28 & 70,90 & 5,19 & 13,66 & 150,50 \\
\hline Itapeaçu & Urucurituba (AM) & $0-20$ & 34,32 & 64,40 & 3,18 & 20,23 & 425,35 \\
\hline Caldeirão & Iranduba (AM) & $0-20$ & 35,33 & 31,30 & 2,23 & 14,02 & 339,61 \\
\hline TP do Limão & Barreirinha (AM) & $0-20$ & 37,99 & 33,70 & 2,28 & 14,80 & 53,89 \\
\hline C. Laranjal & Manacapuru (AM) & $0-10$ & 62,70 & 40,51 & 4,64 & 8,73 & 211,56 \\
\hline Média & & - & 28,49 & 31,64 & 1,94 & 17,37 & 405,55 \\
\hline Mediana & & - & 28,41 & 25,60 & 1,50 & 16,67 & 339,61 \\
\hline Desvio padrão & & - & 13,64 & 20,66 & 1,38 & 4,39 & 293,56 \\
\hline Assimetria & & - & 1,22 & 1,14 & 1,22 & 0,36 & 0,55 \\
\hline Curtose & & - & 0,79 & 0,31 & 0,79 & $-0,22$ & $-0,70$ \\
\hline Shapiro Wilks $\left({ }^{1}\right)$ & & - & $0,94^{\mathrm{NS}}$ & $0,86^{\mathrm{NS}}$ & $0,86^{*}$ & $0,92^{\mathrm{NS}}$ & $0,93^{\mathrm{NS}}$ \\
\hline CV $(\%)$ & & - & 47,87 & 65,29 & 71,16 & 25,28 & 72,39 \\
\hline Neossolo & $\mathrm{P}$, Figueiredo (AM) & $0-20$ & 9,94 & 11,20 & 0,25 & 44,87 & 4,34 \\
\hline Argissolo & R. Preto da Eva (AM) & $0-20$ & 6,23 & 7,60 & 0,50 & 15,19 & 0,62 \\
\hline Cambissolo & Coari (AM) & $0-10$ & 11,06 & 10,30 & 0,65 & 15,84 & 3,10 \\
\hline Latossolo & Manaus (AM) & $0-20$ & 20,82 & 21,40 & 1,72 & 12,45 & 5,58 \\
\hline Média & & - & 12,01 & 29,31 & 0,78 & 22,09 & 3,41 \\
\hline Mediana & & - & 10,50 & 10,75 & 0,58 & 15,52 & 3,72 \\
\hline Desvio padrão & & - & 6,22 & 13,66 & 0,65 & 15,26 & 2,12 \\
\hline CV (\%) & & - & 51,80 & 46,59 & 83,04 & 69,08 & 62,10 \\
\hline
\end{tabular}

* Significativo a $5 \%$ de probabilidade. ${ }^{\text {NS }}$ : Não-significativo. Análises estatísticas realizadas com duas repetições. 

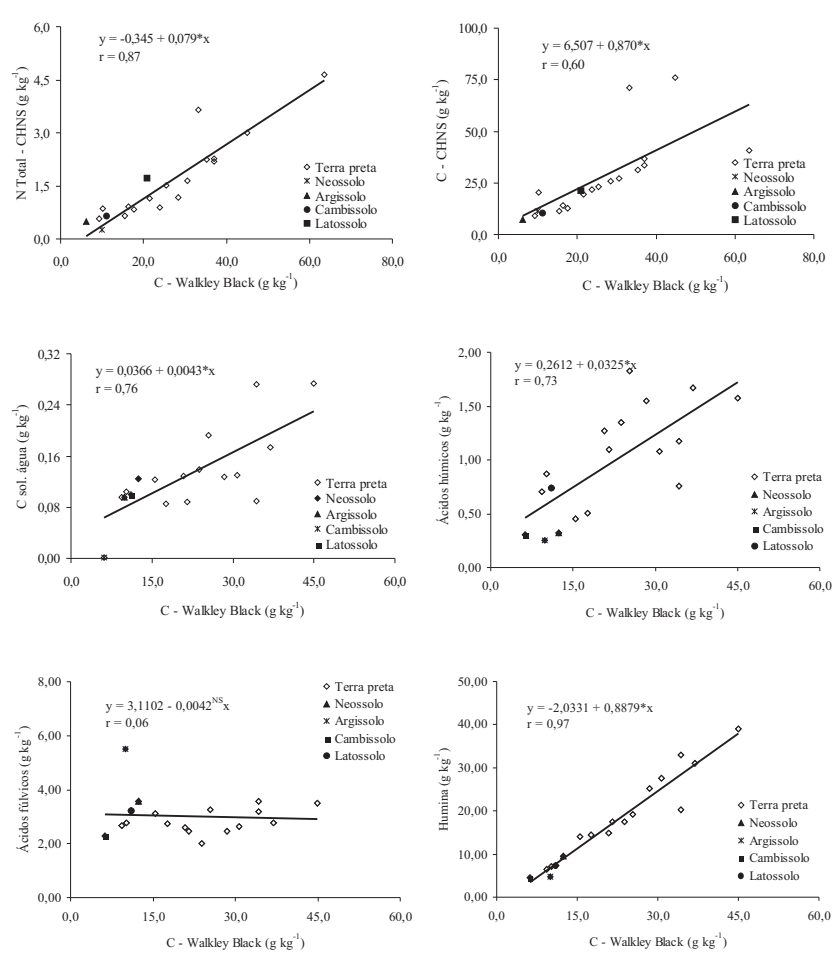

Figura 1. Correlações entre o $C$ (Walkley Black) com $N$ e C (CHNS), C solúvel em água, ácidos fúlvicos, ácidos húmicos e fração humina. *significativo a $5 \%$ de probabilidade. NS: Não significativo. Média das amostras de solo.

Os teores de C, obtidos na TPI pelo WalkleyBlack e pelo analisador CHNS foram, na média dos dois métodos, $194 \%$ maiores que nos dos demais solos representativos da região. ZECH et al. (1990) observaram nos solos antropogênicos materiais originados da matéria orgânica (MOS) na forma estável com alto peso molecular e estruturas de anéis aromáticos unidos, situação diferente da encontrada nos Latossolos, com os polissacarídeos totalmente degradados, sendo essa característica da MOS na terra preta, provavelmente, devida à atividade microbiana do solo. Apesar dessa estabilidade, a relação $\mathrm{C} / \mathrm{N}$ nos solos de TPI foi apenas $20 \%$ maior do que a média do Latossolo, Cambissolo e Argissolo e 158\% menor que no Neossolo (Tabela 2). Os teores de P orgânico indicaram a mesma tendência que o disponível, revelando que o incremento do teor de $\mathrm{P}$ no solo na TPI pode estar diretamente relacionado à ocupação humana nessas localidades.

Nos teores de $C$ determinados por WalkleyBlack e pelo analisador CHNS, houve correlações significativas entre si. Apesar da significância, o Walkley-Black mostrou ser menos sensível na determinação em solos com altos teores de carbono total $\left(>64,0 \mathrm{~g} \mathrm{~kg}^{-1}\right)$ que o analisador CHNS (Figura 1), provavelmente em decorrência da grande quantidade de carbono no solo na forma de carvão (STEINER et al. 2004b), de difícil digestão com ácido sulfúrico concentrado. Segundo SKJEMSTAd e TAYLOR (1999), dependendo do tipo de solo, a eficiência na determinação do carbono por Walkley-Black pode variar de $56 \%$ a $100 \%$, o que não ocorre com o método de oxidação por combustão que converte todo $\mathrm{C}$ em presença do oxigênio para $\mathrm{CO}_{2}$ durante o processo de aquecimento.

O C solúvel em água (CSA) e as substâncias húmicas (CAF, CAH e CHU) na TPI tiveram maior contribuição no C orgânico quando comparado com os solos-referência (Tabela 3), e com exceção do CSA, o $\mathrm{CAF}, \mathrm{CAH}$ e CHU houve distribuição normal, com coeficientes de assimetria e curtose próximos de 0 e 3 . Com base nas características de solubilidade, nos solos antropogênicos, observaram-se maiores quantidades de ácidos húmicos e humina e menor contribuição dos ácidos fúlvicos, com maior relação CAH/CAF $(0,43)$ quando comparados com os outros solos da região $(\mathrm{CAH} / \mathrm{CAF}=0,09)$. A relação mais próxima de 1 verificada nos solos antropogênicos é mais um indicativo da presença de características favoráveis ao húmus, demonstrando a melhor qualidade do solo mesmo em condições favoráveis para rápida mineralização, possivelmente em razão da maior atividade biológica, promovendo a síntese de substâncias mais condensadas (CANELLAs et al., 2001).

$\mathrm{O}$ aumento na quantidade de $\mathrm{CAH}$ nos solos antropogênicos, quando comparados com outras classes de solos (Tabela 3 e Figura 1), pode ser indicador da melhor eficiência na humificação ocasionada pelo aumento da atividade microbiana (STEINER et al., 2004a), responsável pela síntese de substâncias húmicas mais condensadas (CANELlas et al., 2001). Esse incremento na humificação da TPI, quando comparado com os outros solos analisados, também pode ser devido aos altos teores de Ca presente no complexo de troca (Tabela 1), que favorecem as atividades dos anelídeos e térmitas, fornecendo uma matéria orgânica menos solúvel e com agregados mais estáveis (LiMA et al., 2002).

Nos solos estudados, o conteúdo de ácidos fúlvicos independe do teor de matéria orgânica (CAF » $\left.3,1 \mathrm{~g} \mathrm{~kg}^{-1}\right)$. O mesmo não ocorre com o C solúvel em água, ácidos húmicos e humina, cujos coeficientes de correlação são significativos (Figura 1). Os pontos abaixo da reta na equação de regressão obtida entre o carbono solúvel em água e C orgânico indicam em alguns solos de TPI pouco CSA, o inverso que ocorre com as frações de ácidos húmicos e humina. Esses resultados, segundo LimA et al. (2002), são em decorrência do caráter eutrófico dos solos antropogênicos, o que favorece o acúmulo de formas mais estáveis à decomposição da matéria orgânica do solo. 
Tabela 3. Carbono solúvel em água (CSA), ácidos fúlvicos (CAF), ácidos húmicos (AH) e fração humina (CHU) nos solos estudados e estatística descritiva

\begin{tabular}{|c|c|c|c|c|c|c|}
\hline Sítio & Localização & Prof & CSA & CAF & $\mathrm{CAH}$ & $\mathrm{CHU}$ \\
\hline & & $\mathrm{cm}$ & \multicolumn{4}{|c|}{$\mathrm{g} \mathrm{kg}^{-1}$} \\
\hline Rio Urubu & R. Preto da Eva (AM) & $0-10$ & 0,274 & 3,512 & 1,574 & 39,04 \\
\hline Rio Urubu & R. Preto da Eva (AM) & $0-20$ & 0,105 & 2,764 & 0,870 & 7,11 \\
\hline Hatahara & Iranduba (AM) & $0-20$ & 0,130 & 2,643 & 1,079 & 27,49 \\
\hline Hatahara & Iranduba (AM) & $20-40$ & 0,128 & 2,445 & 1,546 & 25,20 \\
\hline Hatahara & Iranduba (AM) & $40-60$ & 0,138 & 2,022 & 1,346 & 17,44 \\
\hline Serra baixa & Manacapuru & $0-20$ & 0,123 & 3,101 & 0,451 & 14,09 \\
\hline Jiquitaia & R. Preto da Eva (AM) & $0-20$ & 0,193 & 3,262 & 1,828 & 19,08 \\
\hline Jiquitaia & R. Preto da Eva (AM) & $20-40$ & 0,174 & 2,767 & 1,671 & 31,05 \\
\hline Açutuba & Iranduba (AM) & $0-20$ & 0,096 & 2,680 & 0,702 & 6,43 \\
\hline Jiquitaia & R. Preto da Eva (AM) & $0-10$ & 0,088 & 2,467 & 1,098 & 17,35 \\
\hline C. Laranjal & Manacapuru (AM) & $0-20$ & 0,085 & 2,748 & 0,506 & 14,42 \\
\hline Caburi & Parintins (AM) & $0-20$ & 0,129 & 2,588 & 1,268 & 14,81 \\
\hline Itapeaçu & Urucurituba (AM) & $0-20$ & 0,090 & 3,552 & 1,170 & 20,31 \\
\hline Caldeirão & Iranduba (AM) & $0-20$ & 0,272 & 3,184 & 0,759 & 32,95 \\
\hline Média & & - & 0,145 & 2,838 & 1,133 & 20,482 \\
\hline Mediana & & - & 0,128 & 2,756 & 1,134 & 18,220 \\
\hline Desvio padrão & & - & 0,063 & 0,433 & 0,435 & 9,568 \\
\hline Assimetria & & - & 1,124 & 0,300 & $-0,085$ & 0,250 \\
\hline Curtose & & - & 0,224 & 0,393 & $-1,160$ & $-0,774$ \\
\hline Shapiro Wilks $\left({ }^{1}\right)$ & & - & $0,84^{*}$ & $0,95^{\mathrm{NS}}$ & $0,97^{\mathrm{NS}}$ & $0,96^{\mathrm{NS}}$ \\
\hline CV (\%) & & - & 43,348 & 15,247 & 38,374 & 46,713 \\
\hline Neossolo & P. Figueiredo (AM) & $0-20$ & 0,125 & 3,583 & 0,319 & 9,43 \\
\hline Argissolo & R. Preto da Eva (AM) & $0-20$ & 0,096 & 5,503 & 0,249 & 4,73 \\
\hline Cambissolo & Coari (AM) & $0-10$ & 0,002 & 2,282 & 0,307 & 4,55 \\
\hline Latossolo & Manaus (AM) & $0-20$ & 0,100 & 3,224 & 0,739 & 7,35 \\
\hline Média & & - & 0,081 & 3,648 & 0,404 & 6,515 \\
\hline Mediana & & - & 0,067 & 2,501 & 0,315 & 4,421 \\
\hline Desvio padrão & & - & 0,054 & 1,353 & 0,226 & 2,328 \\
\hline $\mathrm{CV}(\%)$ & & - & 67,249 & 37,098 & 55,946 & 35,795 \\
\hline
\end{tabular}

$\left({ }^{1}\right) *$ Significativo a $5 \%$ de probabilidade. ${ }^{\text {NS }}$ :Não-significativo. Análises estatísticas realizadas com duas repetições.

\section{CONCLUSÕES}

1. Observaram-se nos sítios de Terra Preta do Índio grande heterogeneidade nos teores de $\mathrm{N}$ total, C orgânico, P disponível e orgânico, K disponível, Ca e Mg trocável, quando comparados com outros solos representativos da região.

2. A ação antrópica advinda da ocupação précolombiana ocasionou a manutenção do conteúdo de ácidos húmicos e humina e diminuição de ácidos fúlvicos, com conseqüente melhoria na qualidade da matéria orgânica do solo.

3. O caráter eutrófico da maioria dos solos antropogênicos contrastam com o Latossolo, Neossolo, Argissolo e Cambissolo, típico da Amazônia.

4. Com o método Walkley-Black houve limitações na determinação do carbono nos solos antropogênicos, quando comparado com analisador CHNS. 


\section{AGRADECIMENTOS}

Aos pesquisadores Wenceslau Geraldes Teixeira e Gilvan Coimbra Martins, da Embrapa Amazônia Ocidental, pelas amostras de solo utilizadas neste trabalho; à bióloga Márcia Pereira de Almeida pelo apoio nas análises e à Embrapa, pelo financiamento através do Macroprograma 2, Projeto "Terra Preta Nova".

\section{REFERENCIAS}

ALVAREZ VENEGAS, V.H.; NOVAIS, R.F.; BARROS, N.F.; CANTARUTTI, R.B.; LOPES, A.S. Interpretação dos resultados das análises de solos. In: RIBEIRO, A.C.; GUIMARÃES, P.T.G.; ALVAREZ VENEGAS, V.H. (Ed.). Recomendação para o uso de corretivos e fertilizantes em Minas Gerais, 5. ${ }^{a}$ Aproximação. Viçosa: CFSEMG, 1999. p.25-32.

CAMARGO, F.A.O.; SANTOS, G.A.; GUERRA, J.G.M. Macromoléculas e substâncias húmicas. In: SANTOS, G.A.; CAMARGO, F.A.O. (Ed.). Fundamentos da matéria orgânica do solo: ecossistemas tropicais e subtropicais. Porto Alegre: Gênesis, 1999. p.27-39.

CANELLAS, L.P.;SANTOS, G.A.; RUMJANEK, V.M.; MORAES, A.A.; GURIDI, F. Distribuição da matéria orgânica e características de ácidos húmicos em solos com adição de resíduos de origem urbana. Pesquisa Agropecuária Brasileira, Brasília, v.36, p.1529-1538, 2001.

COELHO, A.M. Agricultura de precisão: manejo da variabilidade espacial e temporal dos solos e das culturas. In: CURI, N.; MARQUES, J.J.; GUILHERME, L.R.G.; LIMA, J.M.; LOPES, A.S.; ALVAREZ VENEGAS, V.H. (Ed.).Tópicos em Ciência do Solo, Viçosa: SBCS, v.3, p.243-290, 2003.

DEMATTÊ, J.L.I. Manejos de solos ácidos dos trópicos úmidos - Região Amazônica. Campinas: Fundação Cargill, 1988. 215p.

EMBRAPA. Manual de métodos de análise de solo. Rio de Janeiro: CNPS/EMBRAPA, 1997. 212p.

GOMES, F.P.; GARCIA, C.H. Estatística aplicada a experimentos agronômicos e florestais. Piracicaba: FEALQ, 2002. 309p.

KERN, D.C.; KÄMPF, N. Antigos assentamentos indígenas na formação de solos com terra preta arqueológica na região de Oriximiná, Pará. Revista Brasileira de Ciência do Solo, Campinas, v.13, p.219-225, 1989.

LEHMANN, J.; KERN, D.C.; GERMAN, L.A.; McCANN, J.; MARTINS, G.C.; MOREIRA, A. Soil fertility and production potential. In: LEHMANN, J.; KERN, D.C.; GLASER, B.; WOODS, W.I. (Ed.). Amazonian dark earths; origin, properties and management. Dordrecht: Kluwer Academic Publishers, 2003. p.105-124.

LIMA, H.N.; SCHAEFER, C.E.R.; MELLO, J.W.V.; GILKES, R.J.; KER, J.C. Pedogenesis and pre-Colombian land use of "Terra Preta Anthrosols" ("Indian black earth") of Western Amazonia. Geoderma, St Paul, v.110, p.1-17, 2002.
LOPEZ-HERNADEZ, D.; SIEGERT, G.; RODRIGUES, J.V. Competitive adsorption of phosphate with malate and oxalate by tropical soils. Soil Science Society of American Journal, Madison, v.50, p.1460-1462, 1986.

MOREIRA, A.; ALMEIDA, M.P.; COSTA, D.G.; SANTOS, L.S. Acidez potencial pelo método do $\mathrm{pH}$ SMP no Estado do Amazonas. Pesquisa Agropecuária Brasileira, Brasília, v.39, p.89-92, 2004.

MOREIRA, A.; MALAVOLTA, E. Variação das propriedades químicas e físicas do solo e na matéria orgânica em agroecossistemas da Amazônia Ocidental (Amazonas). Piracicaba: CENA/USP, 2002. 79p.

OLSEN, S.R.; SOMMER, L.E. Phosphorus. In: MILLER, R.H.; KENNEY, D.R. (Ed.). Methods of soil analysis. Madison: American Society of Agronomy, 1982. p.403-430.

PRIMAVESI, A. Manejo ecológico do solo. São Paulo: Editora Nobel, 1990. 549p.

RAIJ, B.; QUAGGIO, J.A.; CANTARELLA, H.; ABREU, C.A. Recomendações de adubação e calagem para o Estado de São Paulo. In: RAIJ, B.; CANTARELLA, H.; QUAGGIO, J.A.; FURLANI, A.M.C. (Ed.). Recomendações de adubação e calagem para o Estado de São Paulo, 1997. p.1-13.

RIBEIRO JÚNIOR, P.J. Métodos geoestatísticos no estudo da variabilidade espacial de parâmetros do solo. 1995. 99f. Dissertação (Mestrado em Estatística) - Escola Superior de Agricultura Luiz de Queiroz (USP), Piracicaba.

SCHNITZER, M. Organic matter characterization. In: PAGE, A.L. (Ed.). Methods of soil analysis. Madison: American Society of Agronomy, 1982. p.581-594.

SCHNITZER, M.; KODAMA, H.; RIPMEESTER, J.A. Determination of the aromaticity of humic substances by $\mathrm{X}$ ray diffraction analysis. Soil Science Society of American Journal, Madison, v.55, p.745-750, 1991.

SJOBERG, A. Phosphate analysis of antropic soils. Journal Field Archaeology, Boston, v.3, p.447-454, 1976.

SKEJMSTAD, J.D.; TAYLOR, J.A. Does the Walkley-Black methods determine soil charcoal? Communications Soil Science and Plant Analysis, New York, v.30, p.2299-2310, 1999.

SMITH, N.J.H. Anthrosol and human carrying capacity in Amazonia. Annals of the Association of American Geographers, Durham, v.70, p.553-566, 1980.

STEINER, C.; TEIXEIRA, W.G.; LEHMANN, J.; ZECH, W. Microbial response to charcoal amendments of highly weathered soils and Amazonian dark earths in Central Amazonia - preliminary results. In: GLASER, B.; WOODS, W.I. (Ed.). Amazonian dark earths; explorations in space and time. New York: Springer, 2004. p.195-212a.

STEINER, C.; TEIXEIRA, W.G.; ZECH, W. Slash and char: an alternative to slash and burn practiced in the basin. In: GLASER, B.; WOODS, W.I. (Ed.). Amazonian dark earths; explorations in space and time. New York: Springer, 2004b. p.183-193. 
STEVENSON, F.J. Humus chemistry; genesis, composition, reactions. New York: John Wiley \& Sons, 1994. 496p.

TEIXEIRA, W.G.; MARTINS, G.C. Soil physical characterization. In: LEHMANN, J.; KERN, D.C.; GLASER, B.; WOODS, W.I. (Ed.). Amazonian dark earths; origin, properties and management. Dordrecht: Kluwer Academic Publishers, 2003. p.271-286.

WOODS, W.I. Development of antrosol research. In: LEHMANN, J.; KERN, D.C.; GLASER, B.; WOODS, W.I. (Ed.). Amazonian dark earths; origin, properties and management. Dordrecht: Kluwer Academic Publishers, 2003. p.3-14.
ZECH, W.;HAUMAIER, L.;HEMPFLING, R. Ecological aspects of soil organic matter in tropical land use. In: MAcCARTHY, P.; CLAPP, C.E.; MALCOLM, R.M.; BLOOM, P.R. (Ed.). Humic substances in soil and crop sciences; selected reading. Madison: American Society of Agronomy, 1990. p.187-202. 